\title{
Prevalence of Uropathogen and their Antimicrobial Resistance Pattern in a Tertiary Care Teaching Hospital in Port Blair, India
}

\author{
Abhay Kumar, Amit Banik, Sanjeev H, Agnijeet Palit, Shahina Mustaqim, Manoj Joshi
}

Abhay Kumar, Amit Banik, Sanjeev H, Agnijeet Palit, Shahina Mustaqim, Manoj Joshi

Department of Microbiology, ANIIMS, Port Blair, 744104, Andaman and Nicobar, INDIA.

\section{Correspondence}

Dr. Sanjeev H

Department of Microbiology, ANIIMS,

Port Blair-744104, Andaman and Nicobar

Islands, INDIA.

Mobile no: +91-3192233391

Email: drsanjeevh@gmail.com

History

- Submission Date: 28-01-2017

- Revised Date: 17-07-2017

- Accepted Date: 10-11-2017

\section{DOI : 10.5530/ijmedph.2018.1.8}

Article Available online http://www.ijmedph.org/v8/i1 Copyright

(C) 2018 Phcog.Net. This is an openaccess article distributed under the terms of the Creative Commons Attribution 4.0 International license.

\begin{abstract}
Introduction: Urinary tract infection (UTI) is one of the most common infections seen in the community. Knowledge of common uropathogen and their antimicrobial susceptibility is necessary for initiation of empirical therapy. This study aimed to determine the spectrum of organisms responsible for UTI and their antimicrobial susceptibility pattern in a Medical College Hospital situated in an Island in the Bay of Bengal. Material and Methods: Urine samples received in Microbiology Laboratory between April 2015 to March 2016 were processed according to standard guidelines. The antimicrobial susceptibility testing was performed by the Kirby-Bauer's disc diffusion method and interpreted as per CLSI guidelines. Results: Total of 7850 urine samples were processed during the study period, $956(12.18 \%)$ had significant bacterial growth, of which $65.27 \%$ belonged to women and $34.73 \%$ to men. Gram negative bacteria (GNB) were isolated in $74.79 \%$ followed by Gram Positive bacteria $20.40 \%$ and yeast $4.81 \%$ isolates. The commonest uropathogen was Escherichia coli (48.01\%). The uropathogens were most resistant to Ampicillin (91.5\%), while they were highly sensitive to Levofloxacin and Meropenem. The GPCs were most resistant to Penicillin (66.67\%). Conclusion: Uropathogens were most commonly isolated in 15-30 years age group and the most common isolated microorganism was Escherichia coli. Regular prevalence studies on uropathogens is warranted to assess the trends of the ever changing scenario.

Key words: UTI, Cystitis, Antimicrobial resistance.
\end{abstract}

\section{INTRODUCTION}

Urinary tract infection (UTI) is one of the most common infections seen in the community. ${ }^{1}$ Uncomplicated cystitis in women is the single most common UTI and according to European Association of Urology, is defined as the growth of a single pathogen of $>10^{5}$ colony forming units from properly collected midstream urine sample. ${ }^{2}$ Acute UTI is one of the most important causes of morbidity and has considerable financial implications. It is estimated that 150 million people suffer with UTI every year world-wide, costing the global economy 6 billion dollars. ${ }^{1}$

The higher occurrence of UTI in women is well documented. This is due to the structural anatomy of the female genito-urinary tract, which is more conducive for infection. Further, pregnancy is a physiological state causing increased occurrence of UTI. ${ }^{3,4}$ Other predisposing factors noted are age, sexual activity, diabetes mellitus, immunocompromised states, neurological disorders and catheterisation. Asymptomatic bacteriuria are more prevalent in women with diabetes (26\%) than in women without diabetes $(6 \%)^{.5}$

In children, most often urinary tract infection manifest as fever of unknown origin. Urinary tract infection is seen more frequently in boys during the first year of life. Thereafter, the incidence rate increases in girls compared to boys. ${ }^{6}$
A wide spectrum of organisms are known to cause UTI, commonly encountered being Escherichia coli, Proteus species, Klebsiella species, Enterococcus species and yeasts. ${ }^{7}$ A knowledge of the common uropathogens and their antimicrobial susceptibility is necessary for the initiation of the empirical therapy. ${ }^{8}$

The antimicrobial pattern is diverse and varies with geographical location, influenced by the presence of multi drug resistance (MDR). Multi drug resistance which was limited to hospital setting has been increasingly spreading at the community setting. The factors like overuse and misuse of antimicrobials in allopathic medicine, veterinary and agriculture practise is responsible for the selection of resistant strains and spread of the resistant clones of the organisms. ${ }^{9}$

This study was undertaken to determine the spectrum of organism responsible for UTI and their antimicrobial susceptibility pattern in a medical college hospital located in an Island in the Bay of Bengal.

\section{MATERIAL AND METHOD}

The study was conducted in the Department of Clinical Microbiology of a Medical College Hospital in Port Blair. As per our study design, all urine specimens that were submitted to the laboratory and which were acceptable as per SOP (a clean-catch

Cite this article : Kumar A, Banik A, Sanjeev H, Palit A, Mustaqim S, Joshi M. Prevalence of Uropathogen and their Antimicrobial Resistance Pattern in a Tertiary Care Teaching Hospital in Port Blair, India. Int J Med Public Health. 2018;8(1):38-41. 
Mid-stream urine sample) ${ }^{10}$ of our laboratory, were included for the study. Any other sample other than voided urine was excluded from study (e.g. catheter urine sample). The specimens were screened for uropathogens as per standard guidelines. ${ }^{10}$ In brief, each sample was plated on a Cystine Lactose Electrolyte deficient (CLED) medium using a calibrated standard loop and observed for growth after overnight incubation. A single type of colony with a count of $\geq 10^{5} \mathrm{CFU} / \mathrm{ml}$ was considered significant. Specimen with bacterial count less than $10^{5} \mathrm{CFU} / \mathrm{ml}$ but associated with evidence of inflammation or antibiotic therapy, were further processed as uropathogens. Significant cultures were identified by morphology on Gram's smear, colony characteristics and biochemical reactions as per standard guidelines ${ }^{10}$ and further confirmed by automated identification system (BD Phoenix system, BD, USA).

Antimicrobial susceptibility testing was performed as per Kirby-Bauer disc diffusion method and interpreted as per Clinical and Laboratory Standards Institute (CLSI) guidelines. ${ }^{11}$ The antibiotics tested for the gram positive organism were Penicillin (P, 10U), Ampicillin (AMP, 10 $\mu \mathrm{g}$ ),

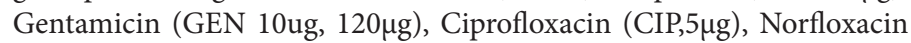

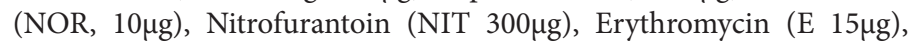

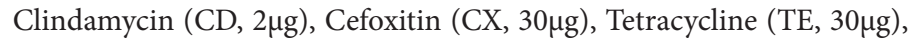

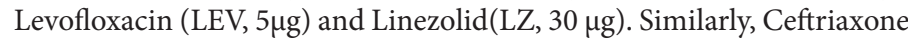
(CTR, $30 \mu \mathrm{g}$ ), Ceftazidime (CAZ, $30 \mu \mathrm{g})$, Cefipime (CPM, $30 \mu \mathrm{g})$, Cefoxitin

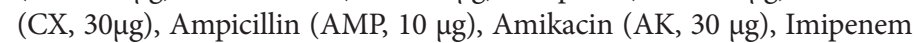
(IMP, $10 \mu \mathrm{g}$ ), Meropenem (MRP,10 $\mu \mathrm{g}$ ), Gentamicin (GEN, $10 \mu \mathrm{g}$ ),

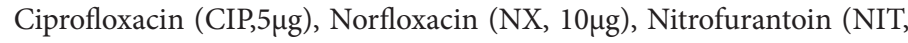
$300 \mu \mathrm{g})$, Cotrimoxazole (COT, 1.25/23.75 $\mu \mathrm{g}$ ) and Piperacillin-tazobactam (PIT, 100/10 $\mu$ g) were tested for gram negative organisms. Methicillin resistance was determined for all Staphylococcus spp. isolates by testing

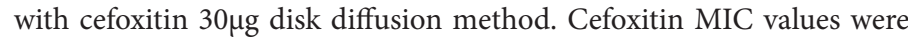
also determined for random isolates $(n=8, \sim 10 \%)$ of MSSA strains. Vancomycin sensitivity to Methicillin Resistant Staphylococcus isolates (MRSA) was determined with MIC interpretative criteria by automated system (BD Phoenix). For Enterococcus spp. Vancomycin disk diffusion was performed. The data was analysed by using Statistical software SPSS Windows version 17.0 (SPSS Inc., Chicago, IL, USA).

\section{RESULT}

A total of 7850 urine specimen were received at the Clinical Microbiology laboratory from April 2015 to March 2016. A significant growth of organism were seen in 956 specimens, which comprised of 624(65.27\%) from females and $332(34.73 \%)$ from males. The mean age of the study group was 36.5years (95\% CI 35.14-38.01years). The age sex distribution is given in Figure 1. The gram negative bacilli $(74.79 \%)$ were the predominant group of organism isolated, of which Escherichia coli (48.01\%) was the commonest (Figure 2). Among the gram positive bacteria, Staphylococcus species comprised of $57.95 \%$. This included 72 isolates (63.7\%) of MSSA, 22 isolates (19.46\%) MRSA and 19 isolates (16.81\%) of CoNS. The antibiogram of commonly isolated uropathogen, Escherichia coli, Klebsiella spp. and Enterococcus spp. is given in Figure 3 and 4 respectively.

\section{DISCUSSION}

Urinary Tract Infection is one of the commonest cause of hospital visit, especially in women of reproductive age group. With emerging MDR in microbes the pathophysiology is often confusing.

The present study evaluated 7850 urine specimen submitted to the Clinical Laboratory over a 12 month period across all age groups (0-76 years). The prevalence of UTI, as determined by a significant positive culture was $12.18 \%$ (956/7850). The prevalence rates documented across the globe ranged from $4.2 \%$ to $97.3 \%$. Such high variations can be attributed to geographical location, climate and local environment, socio-economic status, literacy rates, etc. In a developing nation, such as India, prevalence

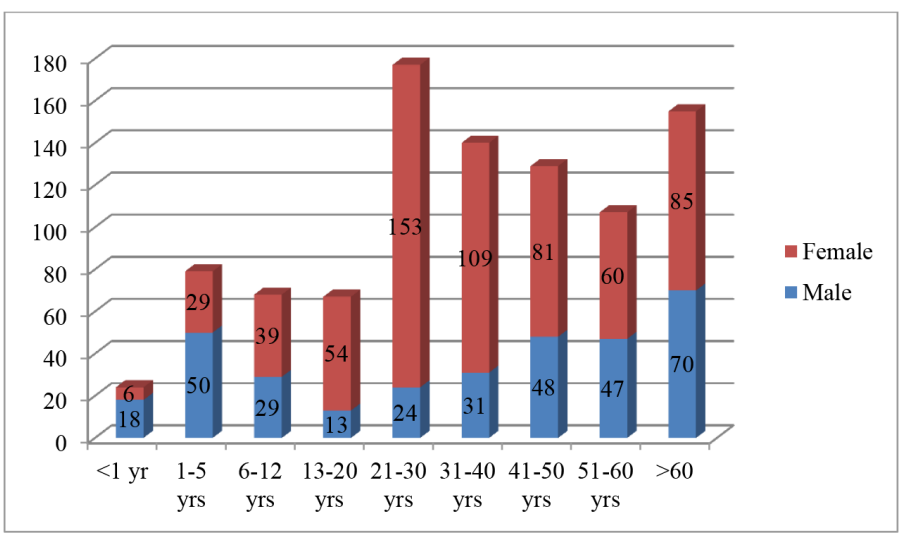

Figure 1: Age - Sex distribution.

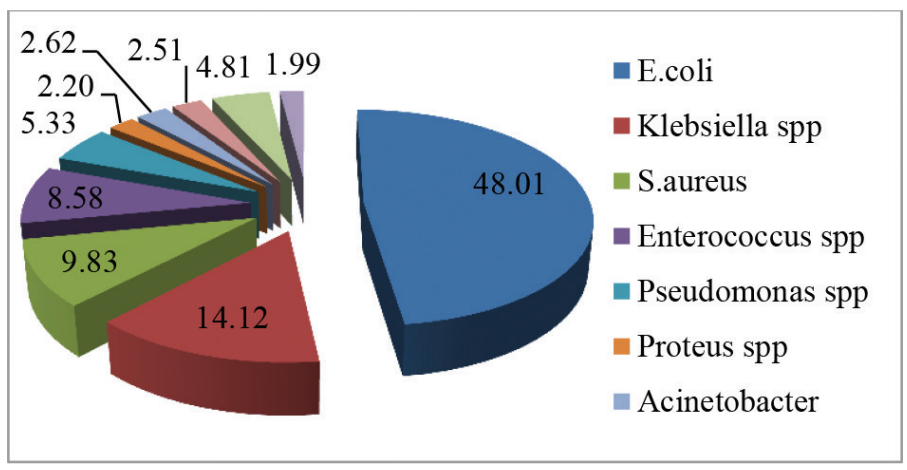

Figure 2: Distribution of organisms isolated $(n=956)$.

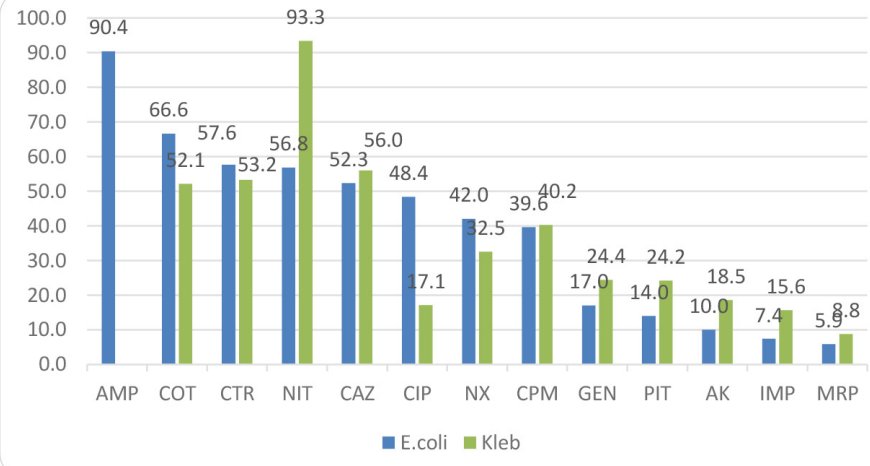

Figure 3: Antimicrobial sensitivity pattern of E.coli $(\mathrm{n}=459)$ and Klebsiella spp. $(n=135)$.

rate ranges from $9.17 \%$ to $36.68 \% .^{3,12,13,14,15}$ The low prevalence in the present study can be explained by a) awareness in the population of the island creating greater emphasis on personal hygiene b) non-specific symptoms being suspected as UTI and submission of urine specimen.

The prevalence rates were higher in women $(65.27 \%)$ as compared to men $(34.73 \%)$ across all age groups. In women, the age group most commonly affected was $21-30$ years $(16.17 \%)$ followed by $31-40$ years (11.52\%). Approximately one half of all UTIs occurred in the reproductive age group (15-45 years). These are well documented findings and attribute the higher occurrence to the anatomical- physiological changes in women during this period. ${ }^{3,16}$ 


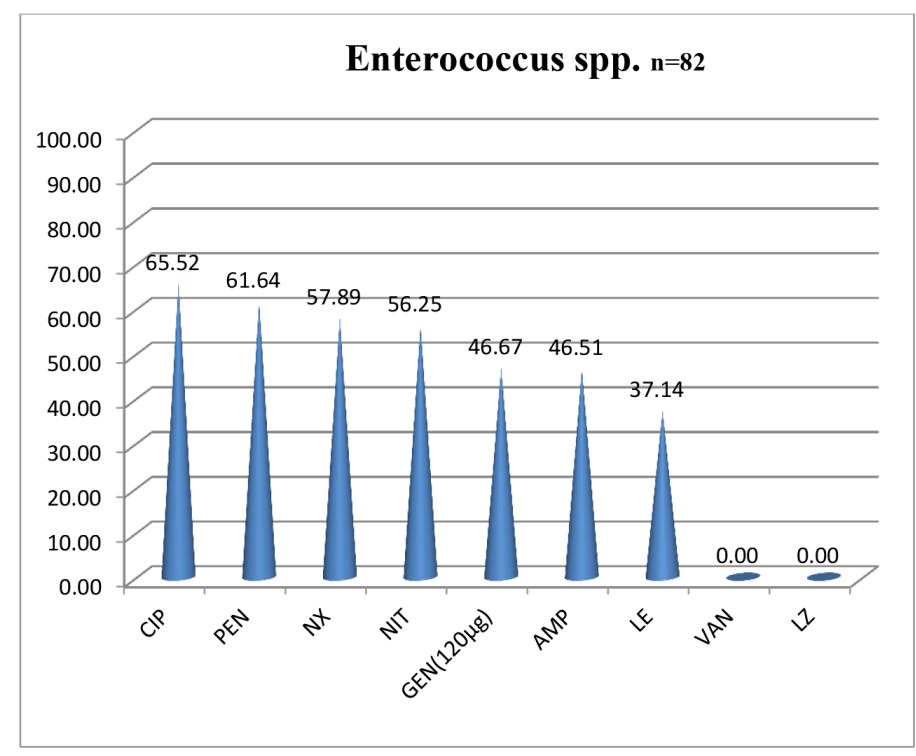

Figure 4: Antimicrobial Resistance pattern of Enterococcus species $(n=82)$.

In contrast, the most common age group affected in men was above 60 years or elderly males (7.4\%). This can be explained as with increasing age men undergo physiological changes such as prostatic enlargement, neurogenic bladder and appearance of co-morbidities such as Type 2 Diabetes mellitus. The above findings of gender prevalence and distribution are in consonance with documented research. Studies have also documented a higher prevalence of UTI in elderly men than in elderly women $^{5,14,16,17}$

Among all urine specimens tested, 171 of them from children had a significant growth on culture. The prevalence between boys to girls was 1.3:1(97:74) across all age group in paediatric population. However, it is interesting to note that the prevalence in children showed progressive reversal in gender with increasing age. The boys to girls proportion rate for UTI were 3:1 in under 1 year age group followed by 1.7:1 in 1-5 year age and 0.7:1 in the 6-12 year age group. Similar findings were reported in other studies which also suggested extensive evaluation of boys as UTI was commonly associated with vesico-ureteric reflux and renal scarring. ${ }^{3,6}$

Gram negative bacilli (74.79\%) were the commonest cause of UTI followed by gram positive cocci (20.40\%) and yeasts (4.81\%). Escherichia coli $(48.01 \%)$ was the most common organism associated with UTI in all age groups and gender, followed by Klebsiella spp. The other gram negative bacteria associated with UTI were Pseudomonas spp (5.33\%), Acinetobacter spp (2.62\%), and Proteus spp. (2.2\%). Gram positive cocci commonly incriminated in UTI were Staphylococcus spp. (11.82\%), followed by Enterococcus spp. (8.58\%).

Antimicrobial susceptibility of gram negative bacteria showed highest resistance to Ampicillin (91.5\%, selected isolates), Cephalosporins (40.96-57.1\%) and least resistance to Aminoglycosides (13.56-20.78\%), Carbapenems (8.01-11.21\%). An interesting finding that was observed was the resistance pattern to Nitrofurantoin in Escherichia coli and Klebsiella spp. The resistance to Nitrofurantoin was 56.8\% in Escherichia coli whereas the resistance was $93.3 \%$ in Klebsiella spp. Similar findings have been noted and reported in earlier studies ${ }^{18 .}$

The most common Gram positive organism incriminated in UTI was Staphylococcus spp. (MSSA, MRSA and CONS) which constituted for $11.82 \%$ followed by Enterococcus spp. (8.58\%). The role of Staphylococcus aureus in causation of ascending UTI is often debated. Isolation of Staphylococcus aureus from urine sample is often secondary to seeding from systemic infection elsewhere. Though Staphylococcus saprophyticus have definite role in UTI, in certain patients Staphylococcus aureus causes ascending urinary tract colonisation and infection. ${ }^{19}$ Cefoxitin MIC values were within acceptable limits of $\leq 2 \mu \mathrm{g} / \mathrm{ml}$ and correlated well with disk diffusion results. Vancomycin MIC values $(<1$ to $2 \mu \mathrm{g} / \mathrm{ml})$ for Staphylococcus spp and disk diffusion values for Enterococcus spp were all within sensitive range, hence proving to be the best drug to fall back upon(Figure 4).

Though a thorough research was made in elucidating the aetiology and antimicrobial susceptibility of uropathogens, confirmation of the mechanism of their antimicrobial resistance could not be performed due to operational reasons and the same is being conducted as an extension of the current study. Another area of limitation of the current study is the deficient clinical data that was available to us for making clinical correlation.

From the above study it has been observed that there is an increase in emergence of multidrug resistant organisms in the community. Therapy of such UTI with MDR organism often becomes difficult due to limited availability of appropriate antibiotics. Such a situation has arisen due to inappropriate use or abuse of antibiotics. Hence, it is necessary that there should be prudent use of available antibiotics.

\section{CONCLUSION}

As evident from the limitation of the present study, we propose that a prospective study on uropathogens with all clinical correlation and study of mechanisms of resistance is very much warranted to understand this problem on a larger perspective.

\section{ACKNOWLEDGEMENT}

Nil

\section{CONFLICT OF INTEREST}

The authors declare that there is tno conflict of interest.

\section{ABBREVIATIONS USED}

UTI: Urinary tract Infection; MDR: Multi drug resistance; SOP: Standard Operating Procedure; CLSI: Clinical and Laboratories standard Institute; MIC: Minimum inhibitory concentration.

\section{SUMMARY}

UTI is most common in women in the age group of 15-30 years and the most common organism incriminated is Escherichia coli. In contrast, the most common age group affected in men is above 60 years of age. However,the prevalence in children showed progressive reversal in gender with increasing age.

\section{REFERENCE}

1. Gonzalez CM, Schaeffer AJ. Treatment of urinary tract infection: whats old, whats new and what works. World J Urol. 1999;6(6):372-82.

2. Linhares I, Raposo T, Rodrigues A, Almeida A. Incidence and Diversity of Antimicrobial Multidrug Resistance Profiles of Uropathogenic Bacteria. BioMed Research International. 2015;1-11.

3. Prakash D, Saxena RS. Distribution and Antimicrobial Susceptibility Pattern of Bacterial Pathogens causing Urinary Tract Infection in Urban Community of Meerut City, India. BioMed Research International. 2013;1-13.

4. Schaeffer AJ, Rajan N, Cao Q, et al. Host pathogenesis in urinary tract infections International Journal of Antimicrobial Agents. 2001;17(4):245-51.

5. Janifer J, Geethalakshmi S, Satyavani K, Viswanathan V. Prevalence of lower urinary tract infection in South Indian type 2 diabetic subjects. Indian J Nephrol. 2009;19(3):107-111.

6. Gupta P, Mandal J, Krishnamurthy S, Barathi D, Pandit N. Profile of urinary tract infections in paediatric patients. Indian J Med Res. 2015;141(4):473-7. 
7. Linhares I, Raposo T, Rodrigues A, Almeida A. Frequency and antimicrobial resistance patterns of bacteria implicated in community urinary tract infections; a ten year surveillance study(2000-2009). BMC Infectious Dis. 2013;13(1):19.

8. Manges AR, Nataraja P, Solberg OD, Dietrich PS, Riley LW. The changing prevalence of drug resistant Escherichia coli clonal groups in a community:evidence for community outbreaks of urinary tract infections. Epidemiol Infect. 2006;134(2):425-31.

9. Furuya EY, Lowy FD. Antimicrobial resistant bacteria in the community setting. Nature Reviews Microbiology. 2006;4(1):36-45

10. Isenberg HD. In Clinical Microbiology Procedures Handbook. Washington DC, ASM. 2007.

11. Performance standards for Antimicrobial susceptibility testing. Clinical and Laboratory Standards Institute M100.CLSI. 2016.

12. Akram M, Shahid M, Khan AU. Aetiology and antibiotic resistance patterns of community -acquired urinary tract infections in JNM C Hospital Aligarh, India. Ann Clin Microbiol Antimicrib. 2007;6(1):4-11.

13. Das RN, Chandrashekhar TS, Joshi HS, Grung M, Shrestha N, Shivananda PG. Frequency and susceptibility profile of pathogens causing urinary tract infections at a tertiary care hospital in western Nepal. Singapore Med J. 2006;47(4):281-5.

14. Sood S, Gupta R. Antibiotic resistance pattern of community acquired uro- pathogens at a tertiary care hospital in Jaipur, Rajasthan. Ind J Com Med. 2012;37(1):39-44.

15. Dash M, Padhi S, Mohanty I, Panda P, Parida B. Antimicrobial resistance in pathogens causing urinary tract infections in a rural community of Odisha, India. J Family Community Med. 2013;20(1):20-6.

16. Ahmed SM, Pai RJ, Koyakutty S, Arya B, Shakir VPA. Urinary tract infections- An overview on prevalence and the antibiogram of Gram Negative Uropathens in a tertiary care centre in North Kerala, India. J Clin Diag Res. 2012;6(7):1192-5.

17. Eshwarappa M, Dosegowda R, Vrithmani IA, Khan MW, Kumar PS, Kempegowda P. Indian J Nephrol. 2011;21:30-36.

18. Sanjeev H, Swathi N, Asha Pai, Rekha R, Vimal K, Ganesh HR. Systematic review of Urinary tract infection caused by Acinetobacter species among hospitalised patients. NUJHS. 2013;3(4):7-9.

19. Muder RR, Brennen C, Rihs JD, Wagener MM, Obman A, Stout JE, et al. Isolation of Staphylococcus aureus from the Urinary tract: Association of isolation with symptomatic urinary tract infection and subsequent Staphylococcal bacterimia. Clin Infect Dis. 2006;42(1):46-50

Cite this article : Kumar A, Banik A, Sanjeev H, Palit A, Mustaqim S, Joshi M. Prevalence of Uropathogen and their Antimicrobial Resistance Pattern in a Tertiary Care Teaching Hospital in Port Blair, India. Int J Med Public Health. 2018;8(1):38-41. 